\title{
Nataša Raschi, Langue française et presse africaine
}

\section{Francesca Piselli}

\section{Q OpenEdition}

\section{Journals}

\section{Edizione digitale}

URL: https://journals.openedition.org/studifrancesi/4843

DOI: $10.4000 /$ studifrancesi.4843

ISSN: 2421-5856

\section{Editore}

Rosenberg \& Sellier

\section{Edizione cartacea}

Data di pubblicazione: 1 avril 2012

Paginazione: 194-195

ISSN: 0039-2944

\section{Notizia bibliografica digitale}

Francesca Piselli, «Nataša Raschi, Langue française et presse africaine», Studi Francesi [Online], 166 (I |

LVI) | 2012, online dal 30 novembre 2015, consultato il 19 novembre 2021. URL: http://

journals.openedition.org/studifrancesi/4843 ; DOI: https://doi.org/10.4000/studifrancesi.4843

Questo documento è stato generato automaticamente il 19 novembre 2021.

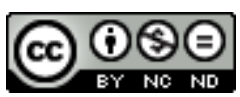

Studi Francesi è distribuita con Licenza Creative Commons Attribuzione - Non commerciale - Non opere derivate 4.0 Internazionale. 


\title{
Nataša Raschi, Langue française et presse africaine
}

\author{
Francesca Piselli
}

\section{NOTIZIA}

NATAŠA RASCHI, Langue française et presse africaine, Roma, Aracne, 2010, pp. 145.

1 Il volume di Nataša Raschi, risultato di una vasta e documentata ricerca sul francese della stampa africana, si segnala come un lavoro innovativo sulla variazione linguistica in ambito francofono. Il corpus di analisi è costituito dallo spoglio dei principali quotidiani di quattro paesi africani (Costa d'Avorio, Burkina Faso, Togo e Benin) nel periodo 2006-2010. L'autrice dedica un capitolo alla lingua della stampa di ciascuno di essi, fornendo dapprima informazioni geografiche, storiche, demolinguistiche e glottopolitiche, per passare poi a un'attenta disamina del lessico e della morfosintassi. L'obiettivo è l'individuazione dei casi più significativi e frequenti in cui i giornalisti africani si discostano dalla norma di riferimento costituita dal cosiddetto français standard. In questo senso la studiosa precisa che «la langue standard n'est plus une langue meilleure que les autres; elle est un point de référence par rapport auquel l'usage de chacun peut être situé» (p. 13). L'approccio usato per la ricerca non è dunque prescrittivo, bensì descrittivo. Raschi sostiene, infatti, che uno degli interessi principali dello studio del francese di oggi risiede proprio nell'analisi delle varianti linguistiche come risultato di una serie di fattori sociali, storici, politici e culturali. In ambito francofono ciò risulta ancora più interessante date le tante componenti, spesso eterogenee e distanti tra loro, che sostanziano questa lingua. Il francese d'Africa è percorso da frizioni, solo apparentemente inconciliabili, tra «ambitions centripètes» (p. 14), ossia l'amore-attrazione per la madrepatria con il desiderio di sentirsi riconosciuti, che si manifestano con fenomeni come quelli dell'ipercorrettismo, e «[ambitions] centrifuges» (ibidem), vale a dire il desiderio di autoaffermazione e di autonomia, come dimostrano le varietà linguistiche affermatesi al di fuori della Francia. 
2 L'indagine sull'aspetto lessicale del francese della stampa di questi quattro paesi dell'ex A.O.F. mostra alcune tendenze comuni, quali la presenza di varianti grafiche, di particolarità lessematiche di termini non attestati in francese, di neologismi - in corso di affermazione nella comunità francofona, legati soprattutto alla politica e creati principalmente per derivazione -, o casi di risemantizzazione di lessemi già esistenti, ma reinterpretati a seconda degli usi locali. I prestiti dalle langues ethniques sono frequenti, soprattutto quando il referente non esiste in francese o quando si vuole sottolineare l'appartenenza a una precisa area geografica. Da notare anche l'uso di expressions figées rimodulate sulla realtà africana, nonché di calchi, rilevati in particolare negli articoli dei quotidiani del Benin e del Burkina Faso. Nel caso della lingua della stampa di quest'ultimo paese, Raschi nota anche fenomeni di ripetizione di due aggettivi con valore differente (ad esempio, «ce grand rassemblement des grands», p. 55), la tendenza ad abusare dell'avverbio «bien», l'uso di acronimi, peraltro sempre accompagnati dalla formula per esteso, oltre che di anglicismi.

3 Sotto il profilo morfosintattico, la ricerca attesta il forte peso dell'oralità nel francese della stampa dei quattro paesi oggetto di studio. Ciò spiega gli scarti dalla norma nell'impiego delle preposizioni, la prevalenza dei rapporti semantici, propria per l'appunto della lingua orale, rispetto a considerazioni di ordine sintattico, la presenza di proposizioni ridondanti - a cui fa da contraltare, però, una spiccata tendenza alla semplificazione, tipica del linguaggio giornalistico, nel quale si fanno notoriamente sentire la pressione della notizia e la necessità di essere il più sintetici possibile -, una certa consuetudine all'anticipazione del complemento oggetto e alla sua ripetizione, finalizzata a mettere in evidenza certi aspetti salienti del discorso. Non vanno dimenticate, inoltre, le deviazioni dalla norma nei passaggi da discorso diretto a discorso indiretto, piuttosto ripetute da parte dei giornalisti del Togo e del Burkina Faso, la massiccia presenza del deittico «-là», in particolare negli articoli sempre di quest'ultimo paese, ma anche della Costa d'Avorio e del Benin, oltre che l'impiego ridondante del pronome «en».

4 Per quanto riguarda il sistema verbale, si osserva un uso piuttosto libero delle nozioni di transitività e intransitività, nel senso che molti verbi sono impiegati indifferentemente secondo l'una o l'altra modalità. I tempi verbali sono usati in maniera spesso incerta; il congiuntivo è il modo che crea più problemi ai giornalisti $\mathrm{e}$ per questo se ne servono solo raramente. In molti articoli del Benin si osserva sia la presenza di alcuni connecteurs non attestati nel francese dell'Hexagone, sia una certa disinvoltura nel loro uso. Altro tratto distintivo della lingua dei giornali di questo paese riguarda la forma negativa: gli articoli mostrano spesso una sovrabbondanza o, viceversa, una totale mancanza di particelle negative.

5 L'approccio linguistico e sociolinguistico adottato dall'autrice ha permesso di accertare che certi fenomeni variazionali, creativi e dinamici sono già profondamente radicati nel contesto linguistico di questi quattro paesi africani. Tali varianti coinvolgono il funzionamento della lingua nella sua globalità, testimoniando un sentimento d'appropriazione completo. Non si può parlare, spiega Raschi, di modifica delle regole, quanto piuttosto d'instabilità del francese d'Africa. Perciò è più corretto riferirsi a quest'ultimo come «langue stratifiée» (p. 117), «langue de la proximité, langue-éponge qui reçoit et qui donne» (ibidem). Si tratta, ovviamente, di un'evoluzione da confermare negli anni a venire. Questo bel volume, redatto con acribia da Nataša Raschi, è corredato da una ricca bibliografia, oltre che da un'aggiornata sitografia, ed è di sicuro 
interesse per lo studio del francese nella sua attualità. Rappresenta un modello di ricerca da applicare in modo sistematico nelle altre aree geografiche della francofonia. 